\title{
Correlation between Percentage of Reticulated Platelets and Heart Score in Patients with Suspected Non-ST Elevation Acute Coronary Syndromes
}

\author{
Fransisca Mulyadi ${ }^{1}$, Delita Prihatni ${ }^{1}$, Coriejati Rita ${ }^{1}$, Dewi Kartika Turbawaty ${ }^{1}$, Astri Astuti ${ }^{2}$ \\ ${ }^{1}$ Department of Clinical Pathology, Faculty of Medicine, Padjadjaran University/Dr. Hasan Sadikin Hospital, Bandung, Indonesia. E-mail: \\ mulyadi.fransisca@gmail.com \\ 2Department of Cardiology and Vascular Medicine, Faculty of Medicine, Padjadjaran University/Dr. Hasan Sadikin Hospital, Bandung, \\ Indonesia
}

\begin{abstract}
Thrombus formation in non-ST Elevation Acute Coronary Syndrome (NSTE-ACS) causes increased platelet consumption, leading to a 20-fold increase of Reticulated Platelets (RP) release. Reticulated platelets have more granules and proteins that make them quickly forming thrombus than mature platelets, potent to form bigger thrombus, and increase the risk of Major Adverse Cardiac Events (MACE). HEART score is a risk stratification for possible NSTE-ACS, which can predict MACE. The study aimed to analyze the correlation between the percentage of reticulated platelets and HEART score. This research was a correlation observational cross-sectional study performed in Dr. Hasan Sadikin Hospital, Bandung, from August 2018 to May 2019. The subjects were patients suspected with NSTE-ACS by clinicians in the Emergency Department of Dr. Hasan Sadikin Hospital. These subjects were assessed for the HEART score and RP percentage. This study involved 52 subjects consisting of a higher number of males (76.9\%) aged 45-64 years old (69.2\%). HEART score stratification in this study was mostly high risk (69.2\%), but none was low risk. Mean of platelet count, absolute $\mathrm{RP}$, and RP percentage were $271 \pm 73 \times 103 / \mathrm{mm}^{3}, 9.3 \pm 4.3 \times 103 / \mathrm{mm}^{3}$, and $3.6 \pm 1.7 \%$, respectively. The correlation test between RP percentage and HEART score with a 95\% confidence interval using Spearman's correlation test showed a significant positive correlation with moderate strength $(p<0.001$ and $r=0.475)$. The percentage of RP in this study was in the normal range. However, there was a significant positive correlation with moderate strength between the percentage of RP and HEART scores in patients with suspected non-ST elevation acute coronary syndrome.
\end{abstract}

Keywords: HEART score, non-ST elevation acute coronary syndrome, percentage of reticulated platelets

\section{INTRODUCTION}

Non-ST Elevation Acute Coronary Syndromes (NSTE-ACS) is described as sudden myocardial ischemia characterized by no persistent ST-segment elevation on ECG. ${ }^{1,2}$ Non-ST elevation acute coronary syndromes are divided into Unstable Angina pectoris (UA), which represents angina with a reversible myocardial injury with normal troponin levels and non-ST Elevation Myocardial Infarction (NSTEMI), which represents angina with an irreversible myocardial injury that leads to elevated troponin levels above the $99^{\text {th }}$ percentile of healthy individuals. ${ }^{1,2}$

In 2016, NSTE-ACS affected $75 \%$ of an estimated 1.1 million patients in the United States, more frequently in the elderly $\geq 65$ years old and less than 45 years old. ${ }^{2-4}$ NSTE-ACS incidence is increasing worldwide, possibly due to smoking, obesity, and underlying diseases, e.g., hypertension and diabetes mellitus, lack of physical activities, fruits, and vegetable consumption. ${ }^{2,4-8}$ Compared to males and post-menopausal females, reproductive-aged females are less likely to have NSTE-ACS due to higher estrogen level that protects them from atherosclerosis manifestations. ${ }^{6,79}$

Non-ST elevation acute coronary syndromes are conditions related to atherosclerosis., ${ }^{2,10,11}$ Atherosclerotic plaque with a thin fibrous capsule, large lipid core, and more macrophages is prone to rupture and cause thrombosis. In contrast, an atherosclerotic plaque with a thick capsule, less lipid core, is more resistant to rupture but could undergo erosion. ${ }^{2,4,12}$ Plaque rupture will expose lipid-rich plaque core to the bloodstream, which leads to adhesion, platelet activation, mediator release (thromboxane A2 and adenosine diphosphate), and platelet migration to the injury area to form 
thrombus that usually causes partial or dynamic occlusion, which differs NSTE-ACS from STEMI. ${ }^{23,5,9,10,12}$

The diagnosis of NSTE-ACS is made by medical history, physical examination, electrocardiography, and troponin level. ${ }^{3,10}$ The diagnosis is often difficult to assess due to atypical chest pain, insignificant abnormal ECG results, or cardiac biomarkers level that takes 4-6 hours post-onset to raise. ${ }^{3,13,14}$ This situation underlies the use of risk stratifications as a step to manage suspected NSTE-ACS., ${ }^{3,14}$ One of the risk stratifications available is HEART score, an acronym for its elements: History, Electrocardiography, Age, Risk factors, Troponin., ${ }^{3,13-16}$ The score is reliable to predict the risk of Major Adverse Cardiac Events (MACE) in 6 weeks after

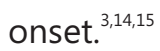

Reticulated Platelets (RP) are newly released from megakaryocytes and still contain Residual Ribonucleic Acid (RNA). ${ }^{17-20}$ Platelets and RP are key players in thrombus formation in NSTE-ACS since RP and their higher potential thrombotic ability will lead to larger and more stable thrombus formation in NSTE-ACS. ${ }^{211,17,18,21,22}$ Hence, RP will increase occlusion and risks of MACE. ${ }^{23}$

\section{METHODS}

This study was a cross-sectional observational research. The subjects were patients suspected with NSTE-ACS in the Emergency Department of Dr. Hasan Sadikin General Hospital. Inclusion criteria were adult patients suspected with NSTE-ACS who had undergone ECG and troponin tests. Exclusion criteria were patients with chronic kidney disease and thrombocytopenia. The sample was whole blood collected in a blood collection tube containing EDTA as an anticoagulant. The sample was used for both troponin I and IPF test. The tubes were immediately inverted 8-10 times and analyzed in automated XN-1000 (Sysmex, Jepang) using PLT-F mode for RP examination and expressed as a percentage (\%) of the total fluorescence platelet count known as Immature Platelet Fraction (IPF). The sample was stable until 8 hours at a temperature of $20^{\circ} \mathrm{C}-26^{\circ} \mathrm{C}$. Troponin levels were measured using a fluorescence immunoassay device Alere Triage Meterpro (Alere, USA).

HEART score was assessed according to its elements. ${ }^{3,15}$ "H" is an acronym of history that describes chest pain. It was divided into a history that was not consistent with chest pain in ACS, varied history that contained traditional and non-traditional elements of ACS, specific history with conventional features of ACS.15 Those were scored 0, 1, 2, respectively. ${ }^{15}$ "E" is the abbreviation for electrocardiography, it was divided into entirely normal ECG, abnormal ECG with repolarization but showed abnormalities lacking significant ST depression, abnormal ECG with significant ST deviation. ${ }^{15}$ Those were scored $0,1,2$, respectively. ${ }^{15}$ "A" represents a patient's age. It was divided into $<45,45-64$, or $>65$ years old. ${ }^{15}$ Those were scored $0,1,2$, respectively. ${ }^{15}$ "R" stands for risk factors consisting of diabetes mellitus, tobacco smoker, hypertension, hypercholesterolemia, obesity, and family history of coronary arterial disease. ${ }^{15}$ This component was divided into no risk factor, 1-2 risk factors, or $>2$ risk factors. ${ }^{15}$ Those were scored $0,1,2$, respectively. ${ }^{15}$ The last one was $T$ as the acronym for troponin level, divided into ? discriminative level, $>1$ - $<3 x$ discriminative level, or $\geq 3$ discriminative levels. 15 Those were scored $0,1,2$, respectively. ${ }^{15}$

The results were accumulated and interpreted for the risk stratification. ${ }^{3,14,15}$ Score $0-3$ was considered as low risk, which showed less possibility of NSTE-ACS. Score 4-6 was considered a moderate risk, which was a potential candidate for observation and further evaluation. A score of 7-10 was regarded as high risk, which needed invasive intervention as soon as possible. ${ }^{3.15}$

Data were analyzed using Statistical Package for the Social Science (SPSS) version 26.0. A normality test was performed on RP data using Kolmogorov Smirnov's test. A correlation test was performed using Spearman's correlation test. The study was approved by the Ethics Committee of Dr. Hasan Sadikin General Hospital (number LB.02.01/X.6.5/ 26/2019) and conducted in Dr. Hasan Sadikin General Hospital from August 2018 to May 2019.

\section{RESULTS AND DISCUSSION}

There were many studies about RP in ACS, including NSTE-ACS. However, this study was the first that analyzed the correlation between RP percentage and HEART score. Fifty-two subjects met the inclusion criteria. The characteristics of the subjects can be seen in Table 1 .

The subjects were mostly male $(76.9 \%)$, age ranged from 37-83 y.o, mostly were 45-64 y.o $(69.2 \%)$, the least was < 45 y.o (7.7\%). This finding was in accordance with references suggesting that NSTE-ACS were less likely to be found in males $<45$ y.o and females < 55 y.o. Similarly, a study by Mirza et al. and Worral-Carter et al. also showed that ACS 
Table 1. Characteristics of subjects $(n=52)$

\begin{tabular}{|c|c|c|c|}
\hline Variable & n (\%) & Mean (SD) & Median (range) \\
\hline \multicolumn{4}{|l|}{ Gender } \\
\hline Male & 40 (76.9) & & \\
\hline Female & $12(23.1)$ & & \\
\hline \multicolumn{4}{|l|}{ Age (years) } \\
\hline$<45$ & $4(7.7)$ & & \\
\hline $45-64$ & $36(69.2)$ & & \\
\hline$\geq 65$ & $12(23.1)$ & & \\
\hline \multicolumn{4}{|l|}{ Risk factors } \\
\hline No risk factors & $2(3.8)$ & & \\
\hline $1-2$ risk factors & $24(46.2)$ & & \\
\hline $\begin{array}{l}\geq 3 \text { risk factors or documented cardiac or } \\
\text { systemic atherosclerotic vascular disease }\end{array}$ & $26(50)$ & & \\
\hline \multicolumn{4}{|l|}{ Chest pain history } \\
\hline Mixed specific \& non-specific chest pain & $15(28.8)$ & & \\
\hline Specific & $37(71.2)$ & & \\
\hline Chest pain onset (hours) & & & $12(4-48)$ \\
\hline \multicolumn{4}{|l|}{ ECG } \\
\hline Normal & $7(13.5)$ & & \\
\hline Non-specific repolarization abnormalities & $18(34.6)$ & & \\
\hline Significant ST-depression & 27 (51.9) & & \\
\hline \multicolumn{4}{|l|}{ Troponin } \\
\hline$\leq$ discriminative level & $6(11.5)$ & & \\
\hline$>1-<3 x$ discriminative level & $4(7.7)$ & & \\
\hline$\geq 3 x$ discriminative level & $42(80.8)$ & & \\
\hline \multicolumn{4}{|l|}{ NSTE-ACS type } \\
\hline UA & $6(11.5)$ & & \\
\hline NSTEMI & $46(88.5)$ & & \\
\hline \multicolumn{4}{|l|}{ Total platelet count $\left(10^{3} / \mathrm{mm}^{3}\right)$} \\
\hline $150-450$ & $51(98.1)$ & $271(73)$ & \\
\hline$>450$ & $1(1.9)$ & & \\
\hline \multicolumn{4}{|l|}{ Absolute reticulated platelets count $\left(10^{3} / \mathrm{mm}^{3}\right)$} \\
\hline $2.5-15.6$ & $49(94.3)$ & $9.3(4.3)$ & \\
\hline$>15.6$ & $3(5.7)$ & & \\
\hline
\end{tabular}

NSTE-ACS: Non-ST Segment Elevation Acute Coronary Syndromes

ECG: electrocardiography, UA: Unstable Angina, NSTEMI: Non-ST elevation myocardial infarction

were more likely to be found in males and only $35 \%$ in females. ${ }^{5,82}$ Those studies also revealed that females were more likely to suffer from ACS in elder age than males. ${ }^{4,24}$ Results in this study followed the theory that all subjects $<45 \mathrm{y}$.o were male, while the youngest female subject aged 49 y.o and had reached menopause. The condition was related to estrogen that regulates lipid metabolism and inflammation system, leading to protective effects to atherosclerotic manifestation including NSTE-ACS. ${ }^{7}$ Menopause, which causes accumulation of visceral fat, central obesity, and increased systolic blood pressure, and risk of metabolic syndrome, e.g., DM and dyslipidemia. ${ }^{\top}$
Most subjects in this study had $>2$ risk factors. The most common risk factors were hypertension and smoking. ${ }^{6}$ This result was following Picariello et al., suggesting that hypertension was the most common risk factor for NSTE-ACS (70-75\%). ${ }^{25}$ Hypertension plays a vital role in NSTE-ACS since it causes endothelial dysfunction, blood flow disturbance, elevated permeability of tunica intima, plaque rupture, and hypertrophy of the left ventricle. ${ }^{25}$ Mirza et al. showed that smoking was the second most common risk factor (62\%). ${ }^{8}$ Smoking produces free radicals causing oxidative stress, which was the primary mechanism of atherosclerosis, inflammation, and platelet activation. ${ }^{9,26}$ 
The most common type of chest pain in this study was the specific type (71.2\%), under the reference, which stated that typical angina was mostly found in NSTE-ACS (80\%). ${ }^{27}$ The atypical type was most likely to be found in elder patients > 75 y.o, female, diabetes patients, which was in accordance with this study. ${ }^{1-4,10,15,27,28}$

In contrast with Grove et al., which revealed that the mean onset of chest pain was 3.6 hours, the onset of chest pain in this study ranged between 4-48 hours with a median of 12 hours. ${ }^{17}$ This might be related to a lack of awareness of the subjects or the former medication to other health facilities since Dr. Hasan Sadikin General Hospital was a tertiary referral hospital.

Electrocardiography results in this study mostly found significant ST-depression (51.9\%). The result was higher than the references, which showed that stated ST-depression was found in $20-25 \%$ cases. $^{28}$ ST-depression and inverted T wave were the most frequent ECG abnormalities found in NSTE-ACS, usually when clinical manifestation appeared., ${ }^{2,10}$ Normal or non-diagnostic ECG results in this study were found in $48.1 \%$ of subjects, consistent with literature that stated that they could be found in $30-50 \%$ cases. ${ }^{1,2,10}$

Non-ST elevation acute coronary syndromes in this study were mostly NSTEMI (88.5\%) with troponin level, mostly $\geq 3 x$ discriminative level (80.8\%). Unstable angina was found in $11.5 \%$ of subjects with HEART score showed moderate risk. The results were according to literature that stated elevated troponin levels had worse prognosis and outcome than normal troponin levels. ${ }^{29}$ This was because troponin level was proportional to irreversible myocardial necrosis area. ${ }^{29}$

HEART score stratification found in this study were mostly high risk (69.2\%), but no low risk was found (Table 2). This fact could be related to chest pain onset, which varied from 4 to 48 hours. Chest pain was a clinical manifestation of myocardial ischemic, which might develop into infarction and caused myocardial necrosis. ${ }^{29}$ The condition would subsequently increase the troponin level and HEART score.

Reticulated platelets are young platelets newly released from megakaryocytes and still contain Residual Ribonucleic Acid (RNA). ${ }^{17-20}$ Studies showed that RNA in RP was used to synthesize granules and glycoproteins (GP), which caused RP to express more granules and glycoproteins, making them more active than mature platelets. ${ }^{17-19,21,22}$ The lifespan of RP in circulation is around 24 hours since the RNA remnants would degrade within 24 hours, and RP turned into mature platelets. ${ }^{21,22}$ In a healthy population, absolute RP count and RP percentage are $2.5-15.6 \times 10^{3} / \mathrm{mm}^{3}$ and $1.0-7.3 \%$, respectively. ${ }^{20}$

Platelets and RP are key players in thrombus formation in NSTE-ACS. ${ }^{2,11,17,18,21,22}$ A study has shown that thrombus formation after plaque disruption increased platelet turnover within the first 6 hours. Bone marrow will respond to acute platelet needs through an alternative mechanism such as a megakaryocyte rupture induced by increased interleukin $1 \alpha(\mathrm{IL}-1 \alpha) .^{30}$ The cytokine is released by damaged endothelial cells and activated platelets, then bind its receptor interleukin-1 receptor (IL-1R). ${ }^{30}$ It stimulates thrombopoiesis by increasing differentiation, maturation of megakaryocytes, and reducing plasma membrane potential, which leads to a weaker membrane structure and triggers megakaryocytes rupture and sheds $\mathrm{RP}^{30}$ This mechanism can provide 20 times more platelets than the proplatelet formation mechanism in normal conditions. ${ }^{30,31}$ Rapid and enhanced RP release into bone marrow vessel occurs primarily within 1 hour. ${ }^{30,31} \mathrm{IL}-1 \alpha$ reaches its peak level in two hours and decreases afterward. ${ }^{32}$ Reticulated platelets have higher thrombotic potential than mature platelets that lead to larger and more stable thrombus formation in NSTE-ACS. ${ }^{2,11,17,1,2,21,22}$ Hence, RP will increase occlusion and risks of MACE. ${ }^{23}$

The percentage of reticulated platelets in this study was mostly in the normal range (99.98\%), with a mean of $3.6 \%$. This result followed a study by Gonzalez-Porras et al. and Grove et al. that revealed mean of RP percentage was $4.6 \%$ and $2.93 \%$, respectively. ${ }^{17,33}$ This normal percentage of reticulated platelets in most subjects might be due to: Onset of chest pain, which varied from 4-48 hours, causing the IL-1 $\alpha$ that induced RP to release to exceed its peak time of 2 hours and replaced by its competitor Interleukin 1-Receptor antagonist (IL-1Ra). ${ }^{32}$; Thrombus formation due to plaque disruption was not massive. Thrombus in NSTE-ACS only caused partial occlusion; therefore, the number of platelets consumed for thrombus formation depended on the size of the thrombus itself and artery lumen. ${ }^{3,5,10,12}$ The number of platelets consumed was parallel with RP released from bone marrow.

One subject had RP percentage $>7.3 \%$. This subject had two histories of myocardial infarction and was still on therapy for CAD. Five other subjects with the highest RP percentage also had a history of myocardial infarction. This result was per literature 
that stated RP percentage was an independent predictor for ACS in CAD patients. ${ }^{18}$

Table 2. HEART score and percentage of RP $(n=52)$

\begin{tabular}{lcc}
\hline \multicolumn{1}{c}{ Variable } & $\mathbf{n}(\%)$ & Mean (SD) \\
\hline HEART score & & \\
$\quad 4-6$ (moderate risk) & $16(30.8)$ & \\
$\quad 7-10$ (high risk) & $36(69.2)$ & \\
$\begin{array}{l}\text { Percentage of } \\
\text { reticulated platelets (\%) }\end{array}$ & & $3.6(1.7)$ \\
$\quad 1-7.3$ & $51(99.98)$ & \\
$>7.3$ & $1(0.02)$ & \\
\hline
\end{tabular}

This study showed a significant positive correlation with moderate strength between RP percentage and HEART score, although most RP was in normal range (Table 3 and Figure 1 ). Unfortunately, there have been no references that correlate RP percentage and HEART score. This study's result was in accordance with the effect of a high RP percentage on the HEART score. High RP percentage in NSTE-ACS showed bone marrow compensation to thrombus formation. ${ }^{14}$ RNA-containing RP could synthesize aggregating protein; hence, it would enhance platelet aggregation and thrombus formation. ${ }^{22,23}$

Nevertheless, this study had limitations. There was no time range limitation for the onset of chest pain. It might be the leading cause of varying RP results. Also, this study did not include the normal

\section{REFERENCES}

1. Roffi M, Patrono C, Collet JP. 2015 ESC guidelines for the management of acute coronary syndromes in patients presenting without persistent ST-segment elevation. Eur Heart J, 2016; 37(1): 267-315.

2. Giugliano RP, Braunwald E. Non-ST elevation acute coronary syndromes. In: Zipes DP, Libby P, Bonow RO, editor. Braunwald's heart disease, a textbook of cardiovascular medicine. $11^{\text {th }}$ Ed., Philadelphia, Elsevier, 2019; 1181-208.

3. Hedayati T, Yadav N, Khanagavi J. Non-ST Segment acute coronary syndromes. Cardiol Clin, 2018; 36: 37-52.

4. Eisen E, Giugliano RP, Braunwald E. Updates on acute coronary syndrome: A review. JAMA Cardiology, 2016; 1(6): 1-13.

5. Timmis A. Acute coronary syndromes. BMJ, 2015; 351: 5153-65.

6. Sanchis-Gomar F, Perez-Quiliz C, Leischik R. Epidemiology of coronary heart disease and acute coronary syndrome. Ann Transl Med, 2016; 4(3): 256-23.

7. Mass AH, Appelman YE. Gender differences in coronary heart disease. Netherlands Heart Journal, 2010; 18(12): 598-603. population to compare the reticulated platelets between NSTEACS and a healthy community.

Table 3. Correlation between percentage of RP and HEART score

\begin{tabular}{ccc}
\hline Variable & r & p \\
\hline $\begin{array}{l}\text { Percentage of RP } \\
\text { and HEART score }\end{array}$ & 0.475 & $<0.001^{*}$ \\
\hline
\end{tabular}

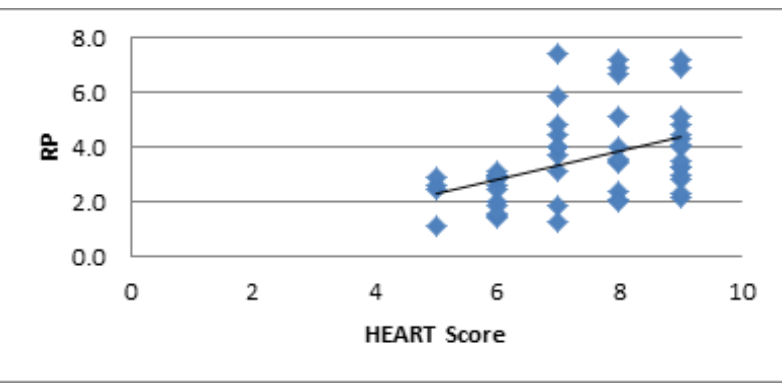

Figure 1. Reticulated platelets distribution to HEART score

\section{CONCLUSION AND SUGGESTION}

This study showed that most RP percentage in NSTE-ACS were normal. However, there was a significant positive correlation with moderate strength between RP percentage and HEART score. Further studies were needed to evaluate the dynamic of RP in NSTE-ACS.

8. Mirza AJ, Taha AY, Khdhir BR. Risk Factors for acute coronary syndrome in patients below the age 40 years. Egypt Heart J, 2018; 70: 233-5.

9. Libby P, Ridker PM, Croce K. Pathophysiology of atherothrombosis. In: Marder VJ, Aird WC, Bennett JS, editor. Hemostasis and thrombosis, basic principles, and clinical practice. Philadelphia, Lippincott Williams \& Wilkins, 2013; 1047-58.

10. Apple FS, Goetze JP, Jaffe AS. Cardiovascular disease. In: Burtis CA, Bruns DE, editor. Tietz fundamentals of clinical chemistry and molecular diagnostics. $7^{\text {th }}$ Ed., Missouri, Elsevier, 2015; 632-49.

11. Abbate R, Cioni G, Ricci I. Thrombosis, and acute coronary syndrome. Thrombosis Research, 2012; 129: 235-40.

12. Bentzon JF, Otsuka F, Virmani R. Mechanisms of plaque formation and rupture. Circ Res, 2014; 114: 1852-66.

13. Backus BE, Six AJ, Kelder JH. Risk scores for patients with chest pain: Evaluation in the Emergency Department. Curr Cardiol Rev, 2011; 7(1): 2-8.

14. Byrne C, Toarta C, Backus B. The HEART score in 
predicting major adverse cardiac events in patients presenting to the Emergency Department with possible acute coronary syndrome: Protocol for a systematic review and meta-analysis. Systematic Reviews, 2018; 7(1): 1-11.

15. Brady W, Souza Kd. The HEART score: A guide to its application in the Emergency Department. Turk J Emerg Med, 2018; 18(1): 47-51.

16. Backus BE, Six AJ, Kelder JC. A Prospective validation of the HEART score for chest pain patients at the Emergency Department. Int J Cardiol, 2013; 168(1): 2153-58.

17. Grove EL, Kristensen SD, Hvas A-M. Immature platelets in patients with acute coronary syndromes. Thromb Haemost, 2009; 101: 151-56.

18. Cesari F, Marcucci R, Gori AM. Reticulated platelets predict cardiovascular death in acute coronary syndrome patients. Thromb Haemost, 2013; 109(5): 1-8.

19. Khalifa KA, Helwa MA, Mohammed AM. Reticulated platelets in acute coronary syndrome patients. Menoufia Med J, 2016; 30: 880-86.

20. Ko YJ, Hur M, Kim H. Reference interval for immature platelet fraction on Sysmex XN hematology analyzer: A comparison study with Sysmex XE-2100. Clin Chem Lab Med, 2014; 1-7.

21. Hoffmann JJ. Reticulated platelets: Analytical aspects and clinical utility. Clin Chem Lab Med, 2014; 52(8): 1107-17.

22. McBane RD, Gonzalez C, Hodge DO. Propensity for young reticulated platelet recruitment into arterial thrombi. J Thromb Thrombolysis, 2013; 1: 1-7.

23. Ibrahim $H$, Schutt RC, Hannawi B. Association of Immature platelets with adverse cardiovascular outcomes. JACC, 2014; 64(20): 2122-29.
24. Worrall-Carter L, McEvedy S, Wilson A. Gender differences in presentation, coronary intervention, and outcomes of acute coronary syndrome patients in Victoria, Australia. Women's Health Issues, 2016; 26(1): 14-20.

25. Picariello C, Lazzeri C, Attana P. The impact of hypertension on patients with acute coronary syndromes. Int J Hypertens, 2011; 1: 1-7.

26. Barua RS, Ambrose JA. Mechanisms of coronary thrombosis in cigarette smoke exposure. Arterioscler Thromb Vasc Biol, 2013; 33: 1460-7.

27. PERKI. Sindrom koroner akut. Pedoman tata laksana sindrom koroner akut. Ed ke-4., 2018; 1-15.

28. Cannon CP, Braunwald E. Non-ST-segment elevation acute coronary syndrome (Non-ST-segment elevation myocardial infarction and unstable angina). In: Loscalzo J, editor. Harrison's Cardiovascular Medicine. $3^{\text {rd }}$ Ed., New York, McGraw-Hill, 2017; 461-68.

29. Jabbar AA, Ahsan C. Troponin I, and the likelihood of hemodynamically significant coronary artery disease in patients with NSTE-ACS. Int J Cardiol, 2013; 170: 17-9.

30. Nishimura S, Nagasaki M, Kunishima S. IL-1a induces thrombopoiesis through megakaryocyte rupture in response to acute platelet needs. J Cell Biol, 2015; 209(3): 453-66.

31. Nieswandt B, Stritt S. Megakaryocyte rupture for acute platelet needs. J Cell Biol, 2015; 209(3): 27-8.

32. Arend WP. The balance between IL-1 and IL-1Ra in disease. Cytokine and Growth Factor Rev, 2002; 13: 323-40.

33. Gonzalez-Porras JR, Martin-Herrero F, Gonzalez-Lopez TJ. The role of immature platelet fraction in acute coronary syndrome. Thromb Haemost, 2010; 103(1): 247-49. 